\title{
Fast Updosing of Subcutaneous House Dust Mite Immunotherapy is Well Tolerated by Pediatric Patients
}

\author{
Martin Glatz', Peter Schmid-Grendelmeier ${ }^{1}$, Alice Koehli2* \\ ${ }^{1}$ Allergy Unit, Department of Dermatology, University and University Hospital Zurich, Switzerland
${ }^{2}$ Division of Allergy, Children's Research Center, University Children's Hospital Zurich, Switzerland
}

*Corresponding author: Alice Koehli, MD, Division of Allergy, Children's Research Center, University Children's Hospital Zurich, Steinwiesstrasse 75, 8032 Zurich, Switzerland, Tel: +41-44-266-7069, E-mail: alice.koehli@kispi.uzh.ch

\begin{abstract}
Data on the safety of a cluster regimen to start a subcutaneous immunotherapy (SCIT) with house dust mite (HDM) extract in pediatric patients are scarce. This study investigates the rate of adverse events (AEs) and the applicability of a cluster regimen for HDM SCIT in pediatric patients, and identifies possible risk factors for the development of AEs. A total of 147 injections with a depot extract ( $D$. pteronyssinus / D. farinae, $50 \%$ each) were applied to $21 \mathrm{HDM}$-sensitized pediatric patients (age 5-18 years) with allergic asthma and/or allergic rhinitis within 2 weeks. All patients completed the cluster regimen with the full cummulative dose. Systemic adverse events (AEs) were mild to moderate (rhinitis nausea or mild asthma), and occurred after 5 injections $(3.4 \%)$ in three different patients (14.3\%). Local AEs occurred in 14 patients (66.7\%); 11 patients had minor local AEs and only 3 patients had major local AEs of a diameter $\geq 5 \mathrm{~cm}$. All AEs developed within 60 minutes after the last injection. Patients with local AEs had higher total IgE serum levels than patients without AEs $(p=0.02)$. In conclusion, the cluster regimen for induction of HDM SCIT is a safe and well tolerated procedure in children.
\end{abstract}

\section{Keywords}

Dust mite sensitization, Subcutaneous immunotherapy, Cluster regimen, Adverse events, Pediatric patients

\section{Introduction}

Allergen Immunotherapy (AIT) is the repeated administration of allergens to an allergic patient. It is the only disease-modifying therapy for allergies, and prevents the progression of allergic rhinitis to asthma in children [1,2]. In conventional Subcutaneous Immunotherapy (SCIT), allergic patients receive increasing allergen doses every few days during a build-up phase of a few months until a therapeutic maintenance dose, which is then applied every 4-6 weeks. Owing the need of frequent physician consultations and many injections within a short period of time, this build-up phase is inconvenient for patients [3], and the main reason not to start or to discontinue SCIT [4]. Cluster regimens with a few injections on each treatment day provide a faster build-up phase are therefore desirable to improve patient's adherence, and may not necessarily have a higher risk of adverse events (AEs) than conventional updosing regimens [25]. Cluster and even rush regimens have been introduced into clinical routine for hymenoptera venom SCIT [6], but are less commonly used for aeroallergens such as house dust mites (HDM) [2] despite a safety profile comparable to conventional SCIT $[3,7,8]$. Cluster regimens for HDM SCIT have mainly been investigated in adult or mixed adult and pediatric study populations [3,8-12], while data on pediatric populations are rarer $[7,13,14]$. We therefore carried out this study to assess the safety and tolerability of a cluster regimen for HDM SCIT in a pediatric population, to assess its applicability in the clinical routine of a pediatric hospital, and to identify risk factors for the development of AEs during the cluster regimen.

\section{Materials and Methods}

\section{Patients}

This is a single-center, retrospective, investigator-initiated study, conducted according to Declaration of Helsinki Principles at the Division of Allergy, Children's Research Center, University Children's Hospital Zurich, Switzerland. All patients were seen between 2006 and 2013 for the duration of the cluster regimen. All patients received the maintenance doses from their pediatrician. The retrospective use of clinical data was approved by the ethical committee of the Canton of Zurich. All patients and and/or legal guardian gave written informed consent for retrospective data analysis. Inclusion criteria were: (i) Age of patient $\leq 18$ years; (ii) Sensitization to HDM (Dermatophagoides pteronyssinus and/or D. farinae) as determined by a positive skin prick test and/or detection of allergen-specific serum IgE antibodies; (iii) Presence of allergic asthma and/or allergic perennial rhinitis; (iv) No previous AIT with HDM within the last 12 months and no concurrent AIT with another allergen; (v) Written informed consent signed by the patient and/or legal guardian.

We recorded the following metadata: sex of patients, age at onset of cluster regimen, forced expiratory volume in 1 second (FEV1), mid expiratory flow between $25 \%$ and $75 \%$ of expired volume (MEF25/75) and fractional exhaled nitric oxide (FeNO). Only FEV1, MEF25/75 and $\mathrm{FeNO}$ values determined at day 0 of the cluster regimen before the first injection were included in the analysis. We determined total serum IgE antibodies, and sensitization to perennial and seasonal allergens 


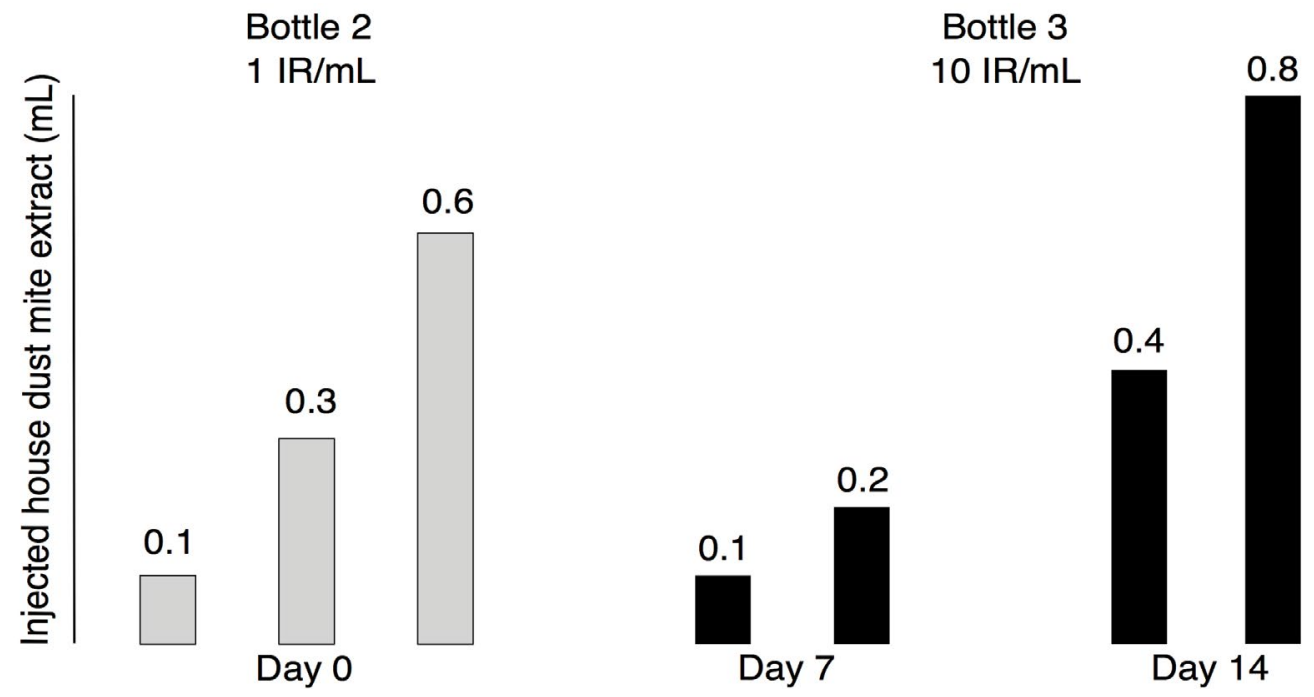

Figure 1: Administration schedule of the cluster regimen. Grey bars indicate injections with house dust mite extract from extract bottle 2 at day 0 . Black bars indicate administration of house dust mite extract from extract bottle 3 at days 7 and 14. Height of bars indicate amount of extract injected, the numbers above the bars indicate the injected amount of extract in $\mathrm{mL}$.

(D. pteronyssinus and D. farinae, Alternaria alternata, cat dander, dog dander, birch pollen, grass pollen) by skin prick test with standardized allergen extracts (Soluprick SQ, ALK, Volketswil, Switzerland) and/ or by detection of allergen-specific serum IgE antibodies using the ImmunoCAP (Thermo Fisher Scientific - Uppsala, Sweden).

\section{Allergen immunotherapy}

All patients received a depot extract adsorbed to calcium phosphate (D. pteronyssinus/D. farinae 50\% each; Phostal, Stallergènes, Dietlikon, Switzerland), injected subcutaneously into the dorsal upper arm in a cluster regimen at days 0,7 and 14 as previously described in adults (Figure 1) [9]. Allergen concentrations in extracts were defined as a reactivity index (IR) of 1 or 10 figure 1 , where 100 IR were defined as the allergen concentration eliciting a mean wheal diameter of $7 \mathrm{~mm}$ in a skin prick test in $30 \mathrm{HDM}$-sensitized individuals. For injections at day 0 , all patients admitted the intermediate care unit of our hospital, received an i.v. line for security reasons and were monitored for 3 hours after the last injection. Injections at days 7 and 14 were applied at the regular outpatient clinic, and patients were monitored for 60 minutes after the last injection. Local AEs with a diameter $<5 \mathrm{~cm}$ were graded as "minor", those with a diameter $\geq 5 \mathrm{~cm}$ as "major" [15]. Systemic AEs were graded according the World Allergy Organization [16]. All patients received the maintenance therapy with HDM extract every 4 weeks according manufacturers' instructions from their local pediatrician.

\section{Statistics}

Analysis was performed with $\mathrm{R}$ software version 3.2.1 [17] Outliers of metadata were visualized with $\mathrm{Q}-\mathrm{Q}$ plots. Continuous metadata were treated non-parametric due to small sample size and presented as median and interquartile range. The rate of AEs in correlation to categorical metadata was tested by Fisher's exact test, in correlation to continuous metadata by Wilcoxon rank test. P values were adjusted for multiple comparison by Benjamini Hochberg correction [18]. Statistical significance for all tests was ascribed to a two-sided alpha level of the adjusted $P$-values $<0.05$, and by $95 \%$ confidence intervals for continuous metadata.

\section{Results}

\section{Patients}

Twenty-one pediatric patients were included in this study (Table 1). Twenty patients (95.2\%) had allergic asthma. Asthma was well controlled in all patients at onset of the cluster regimen, as defined by the Global Initiative for Asthma [19]. In all of the patients that received a lung function within 1 month before onset of the cluster regimen, the FEV1\% was above $80 \%$ of the predicted value, but half of the tested patients showed hints to bronchial inflammation (FeNO $>20 \mathrm{ppb})$. Eighteen of
Table 1: Patient demographics and clinical data.

\begin{tabular}{|l|l|}
\hline Age (years), median (IQR) & $11.2(10-15.3)$ \\
\hline Sex (\% Male) & 66.7 \\
\hline Asthma, $n$ (\%) & $20(95.2)$ \\
Lung function ${ }^{\mathrm{a}}$, n (\% of asthma patients) & $8(40)$ \\
FEV1\%, median of predicted value (IQR) & $93(85.3-125)$ \\
MEF25-75 (I/s), median (IQR) & $68.5(64.8-99.8)$ \\
Median FeNO (ppb), median (IQR) & $18.5(7.8-27.1)$ \\
Treatment, n (\% of asthma patients) & \\
CS + BD + LA & $3(15)$ \\
CS + BD & $13(65)$ \\
CS & $1(5)$ \\
BD & $1(5)$ \\
No treatment & $1(5)$ \\
Data not available & $2(10 \%)$ \\
\hline Allergic rhinitis, $n$ (\%) & $7(33.3)$ \\
\hline Atopic dermatitis, $n$ (\%) & $3(14.3)$ \\
\hline Sensitization to allergens, n/tested (\%) & \\
D. pteronyssinus/D. farinae & $21 / 21(100)$ \\
Alternaria & $1 / 15(6.7)$ \\
Cat dander & $11 / 19(57.9)$ \\
Dog dander & $8 / 15(53.3)$ \\
Birch pollen & $4 / 18(22.2)$ \\
Grass pollen & $8 / 18(44.4)$ \\
\hline
\end{tabular}

aLung functions performed within 1 month before onset of cluster regimen.

Note: BD: bronchodilator; CS: corticosteroid; FeNO: fractional exhaled nitric oxide; FEV1: forced expiratory volume in 1 second; IQR: interquartile range; LA: leukotriene antagonist; MEF25/75: mid expiratory flow between $25 \%$ and $75 \%$ of expired volume.

the asthma patients (90\%) used inhalation therapies at onset of the cluster regimen. The only patient without asthma was an 18-years-old girl with perennial allergic rhinitis, who therefore qualified for SCIT. All patients were sensitized to either both $\operatorname{HDM}(\mathrm{n}=20,95.2 \%)$ or to D. pteronyssinus alone $(\mathrm{n}=1,4.8 \%)$. Of 16 patients tested for HDM with Immuno CAP (76.2\%), 2 patients (12.5\%) had HDM-specific IgE antibody serum titers of CAP class 3, 6 patients (37.5\%) CAP class 4, 4 patients (25\%) CAP class 5 , and 4 patients (25\%) CAP class 6.

\section{Adverse events during cluster regimen}

All patients completed the cluster regimen and received the full cumulative dose. We applied a total of 147 injections during the cluster regimen. All AEs occurred within 60 minutes after the last injection. The majority of AEs comprised minor local AEs (rash, swelling, pruritus) of $<5 \mathrm{~cm}$ in diameter at injection sites, observed in 11 patients (52.4\%) (Figure 2). 


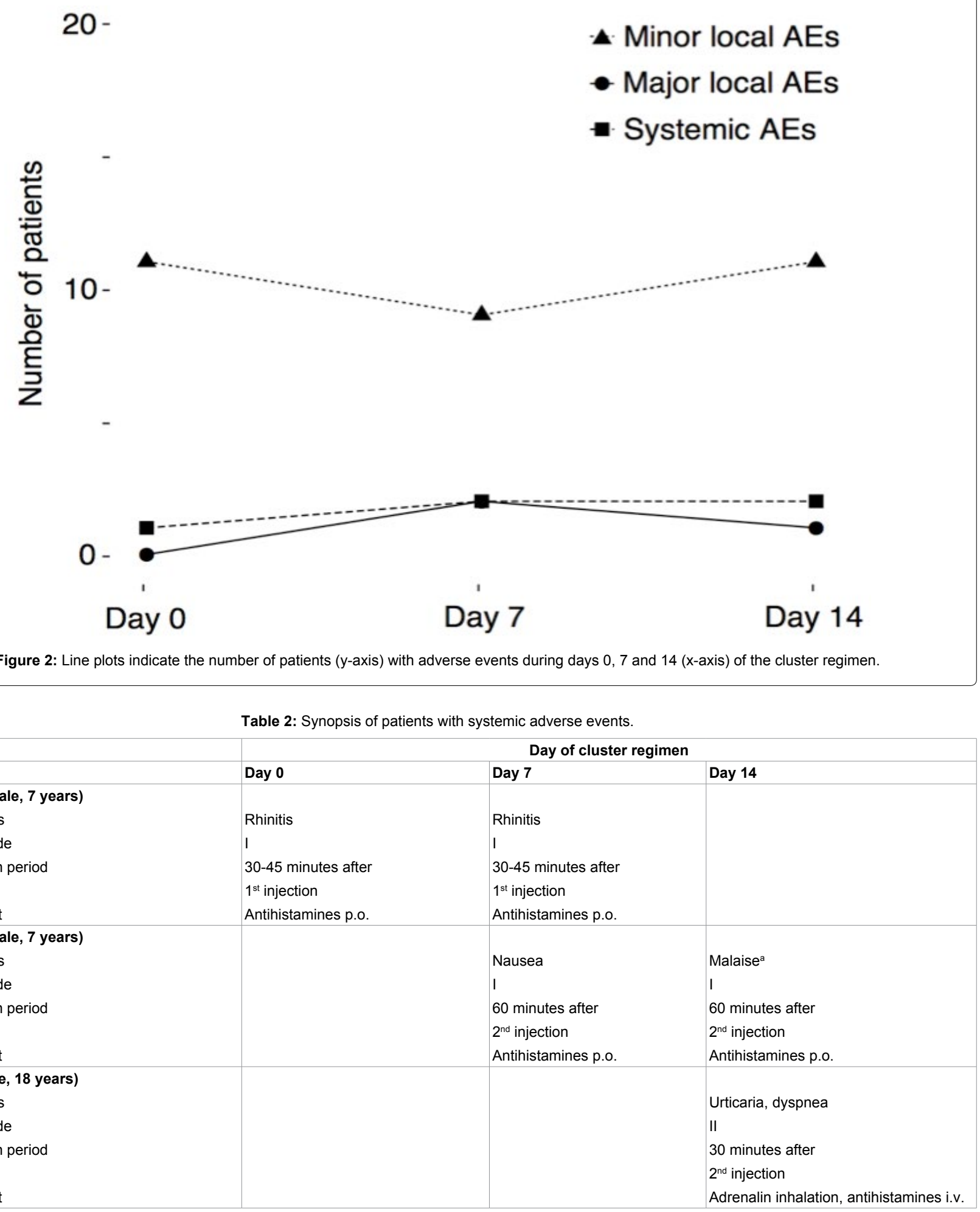

Three patients (14.3\%) experienced major local AEs of $\geq 5 \mathrm{~cm}$ in diameter. Major local AEs did not occur during day 0 of the cluster regimen figure 2, although this was the day with the fastest allergen dose increase and the highest cumulative allergen dose (Figure 1). Two patients had major local AEs at day 7. One was a 15-years-old boy with a $5 \times 5 \mathrm{~cm}$ red swelling, no AEs at day 0 and a minor local $\mathrm{AE}$ at day $14(8 \times 8 \mathrm{~mm}$ red swelling $)$. The other patient with a major local AE at day 7 was a 7 -years-old girl with a $6 \times 6 \mathrm{~cm}$ red swelling. She had minor local AEs at day $0(2 \times 2 \mathrm{~cm}$ red swelling $)$ and day $14(8 \times 8 \mathrm{~mm}$ red swelling). Major local AEs at day 14 occurred in a 12 -years-old boy as a $5 \times 5 \mathrm{~cm}$ swelling at both injections sites, but he had no AEs at days 0 and 7.

Systemic AEs were mild to moderate and observed after 5 injections (3.5\%) in 3 patients (14.3\%). A synopsis of the patients with systemic AEs is depicted in (Table 2). It is debatable if the malaise of patient 2 (a 7-years-old girl) at day 14 was indeed caused by SCIT. The patient was very nervous before the injections and also developed a viral infection of the upper respiratory tract with sore throat and headache shortly after day 14 , both of which may explain the malaise beforehand. She also had minor local AEs with local pruritus at all three days of the cluster regimen. The third patient (an 18-years-old adolescent) who developed a grade II reaction at day 14 had previously received conventional SCIT with HDM for 3.5 years. This SCIT was abrogated 13 months before onset of our cluster regimen due to grade III AEs with urticaria and severe asthma. Owing this history and systemic AEs at the last day of our cluster regimen, he received a halved maintenance dose of $0.4 \mathrm{~mL}$ every 4 weeks after the cluster regimen, which was well tolerated during the whole maintenance phase. Notably, only one patient developed a mild (grade I) systemic $\mathrm{AE}$ at day 0 of our cluster regimen, although this was the day with the fastest increase of the allergen doses (Figure 1). 


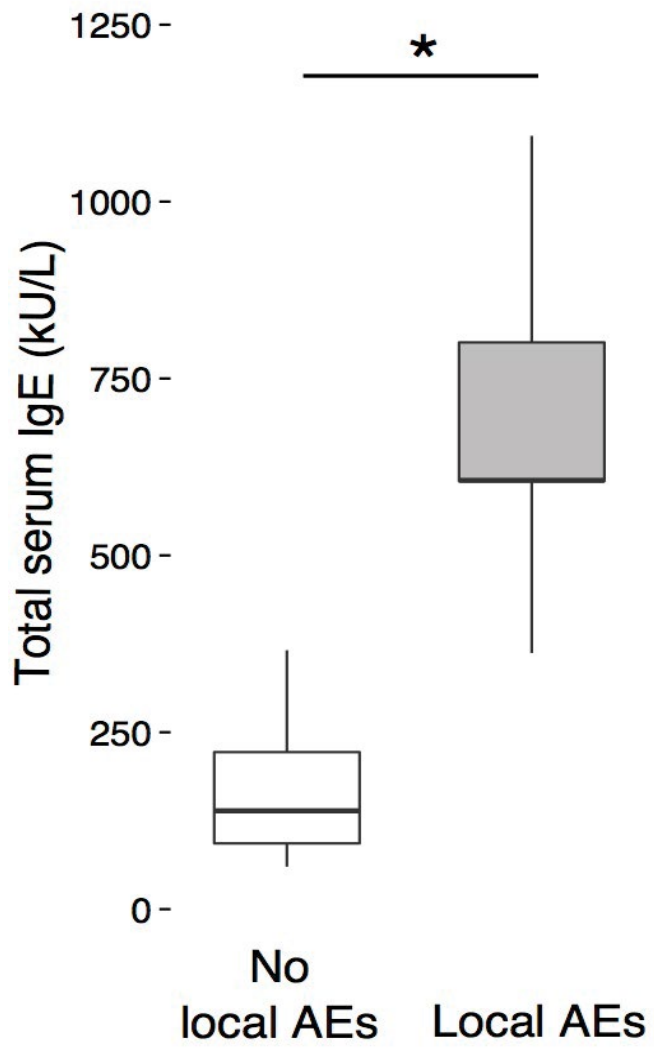

Figure 3: Boxplots indicate total serum lgE levels (y-axis) in patients with local adverse events (AEs) compared to patients with no local AEs.

*indicates $P<0.05$ determined by Wilcoxon rank test.

\section{Risk factors for adverse events}

Patients with mild local AEs tended to have higher total IgE serum levels than children without AEs, but the difference was not significant $(\mathrm{p}=0.2)$. When patients with minor and major local AEs were grouped together, they had significantly higher total IgE serum levels than children without local AEs ( $\mathrm{p}=0.03,95 \% \mathrm{CI}[188$ - 989]) (Figure 3). This indicates that higher serum levels of total IgE might be a risk factor for the development of local AEs during SCIT with HDM. No other clinical or laboratory metadata, particularly not preexisting asthma, correlated with the development of local or systemic AEs.

\section{Discussion}

This study retrospectively assessed the safety and applicability of a cluster regimen in clinical routine for SCIT with HDM in a pediatric population of 21 patients with allergic asthma and/or allergic rhinitis. In the vast majority, AEs were mild and localized with a red swelling of less than $5 \mathrm{~cm}$ in diameter at injection sites, which is considered as common during SCIT and clinically not relevant [1-15]. Systemic AEs and major local AEs were usually mild and occurred in 3 patients $(14.3 \%)$ each. They were as rare as in adults undergoing the same cluster regimen (15\%) [9], resolved with systemic antihistamines or, in one adolescent with adrenalin inhalation, and did not lead to abrogation of the cluster regimen in any case. These results indicate a comparable rate of local and systemic AEs in pediatric (our study) and adult patients [9], and that it is well tolerated by patients of a wide age range.

In other cluster regimens for HDM SCIT in pediatric and adult populations, between 2.4 and $54.2 \%$ of patients had local AEs and between 0 and $3 \%$ of patients had systemic AEs $[3,8,12-14]$. This variability in the rate of AEs may owe to heterogeneity in patient demographics (e.g. age), to different injection schemes, and to different allergen compositions and allergen concentrations of the extracts. For example, a study on 77 children younger than 5 years-of-age [13] reported local and systemic AEs in 3.9\% and 1.3\% of children, respectively, which appears lower than in our study. However, those children were younger (maximum 5 years) than our patients (maximum 18 years), received only 4 injections ( 7 injections in our study), and received an allergen extract exclusively containing D. pteronyssinus (D. pteronyssinus/D. farinae $50 \%$ in our study) from another manufacturer than in our study. Younger individuals have a less pronounced immune response to certain antigens $[20,21]$ and a lower likelihood for systemic AEs in SCIT than older individuals $[22,23]$. Also, an extract exclusively containing $D$. farinae may cause less AEs in HDM sensitized individuals than an extract containing both HDM. Finally, it is difficult to exactly define the allergen concentration of an extract, and it can significantly vary between different manufacturers or even between different batches from the same manufacturer (personal communication with Stallergenes). These factors may contribute to the lower rate of AEs reported by Hernandez et al. [13] than in our study. It therefore appears difficult to compare the rate of AEs between studies using different cluster regimens with different allergen extracts.

The secondary aim of our study was to identify risk factors for the development of AEs during the cluster regimen. We found that higher levels of total IgE in serum are associated with the development of local AEs. A positive correlation between the rate of systemic AEs and serum levels of total and HDM-specific IgE were reported in a Chinese study on $234 \mathrm{HDM}$-sensitized patients undergoing SCIT [24]. However, these data were published in a Chinese journal and essential information such as on the age of the study population was not available from the English abstract. Positive correlations between the rate of AEs and IgE antibodies have been described for cow's milkallergic children undergoing oral AIT [25]. However, the significance for our findings remains unclear, because oral AIT in food allergies is usually accompanied by a higher rate of AEs [1].

We acknowledge several limitations of this study. First, this is not a randomized placebo-controlled trial. However, the purpose of our study was not to compare our cluster regimen with other cluster regimens or its efficacy with placebo. The primary aim of this study was to assess the safety and applicability of cluster regimen in the clinical routine and under real life conditions of a pediatric hospital unit. Second, our study population comprised a limited number of patients $(n=21)$, and the number of patients with major local $(n=$ 3 ) or systemic AEs $(n=3)$ was not big enough for a reliable statistical analysis. However, the size of our study population is comparable to previously published studies on HDM cluster regimens, comprising $15-29$ patients $[3,7,8,11]$. Because the cluster regimen used in our study has already been assessed for its safety and applicability in 47 adult patients [9] and the rate of AEs in pediatric and adult patients is similar, the size of our study population appears sufficient. Some other studies on HDM cluster regimens included a higher number of patients $(\mathrm{n}=40-343)[8,10,12,14]$, but comprised a more heterogeneous study population of pediatric and adult patients and did not adjust statistical analyzes for different age groups $[8,10,12]$, or for different cluster regimens [14].

The cluster regimen used in our study is advantageous particularly for pediatric patients. It comprises only 7 injections within 2 weeks and therefore needs fewer injections and is faster than most other regimens ranging from 8 injections during 3 weeks to 16 injections during 6 weeks [3,7,8,10-12,26]. A fast cluster regimen with few injections is desirable for children and their parents. In our experience, parents usually choose the faster cluster regimen, because it requires less physician visits and therefore keeps their absences of work and those of their children of school to a minimum. Also, young patients usually do not like many injections. Hence, the cluster regimen introduced here might improve adherence of pediatric patients and their parents to SCIT. Adherence to SCIT is of particular importance, because type 1 regulatory $\mathrm{T}$ cells, which are crucial for the development of allergen tolerance, increase in the blood of pediatric patients (8-13 years) after 1 year of HDM SCIT [27].

In conclusion, the cluster regimen presented in this study seems to be safe for pediatric patients with allergic asthma and rhinitis, and 
well applicable in clinical routine. AEs during this cluster regimen for updosing HDM SCIT were usually localized and mild. High total IgE serum levels might indicate a higher risk for local AEs. Because patients reach the maintenance phase of SCIT faster than with other cluster regimens, it may be a useful approach to improve also the adherence of patients to treatment.

\section{Ethical Statement}

The retrospective assessment and analysis of clinical data was approved by the ethical committee of the Canton of Zurich.

\section{References}

1. Jutel M, Agache I, Bonini S, Burks AW, Calderon M, et al. (2015) Internationa consensus on allergy immunotherapy. J Allergy Clin Immunol 136: 556-568.

2. Pfaar O, Bachert C, Bufe A, Buhl R, Ebner C, et al. (2014) Guideline on allergen-specific immunotherapy in ige-mediated allergic diseases. Allergo J Int 23: 282-319.

3. Mauro M, Russello M, Alesina R, Sillano V, Alessandrini A, et al. (2006) Safety and pharmacoeconomics of a cluster administration of mite immunotherapy compared to the traditional one. Eur Ann Allergy Clin Immunol 38: 31-34.

4. Cox $L$ (2006) Accelerated immunotherapy schedules: Review of efficacy and safety. Ann Allergy Asthma Immunol 97: 126-137.

5. Quiralte J, Justicia JL, Cardona V, Davila I, Moreno E, et al. (2013) Is faster safer? Cluster versus short conventional subcutaneous allergen immunotherapy. Immunotherapy 5: 1295-1303.

6. Kosnik M, Korosec P (2015) Venom immunotherapy: Clinical efficacy, safety and contraindications. Expert Rev Clin Immunol 11: 877-884.

7. Schubert R, Eickmeier O, Garn H, Baer PC, Mueller T, et al. (2009) Safety and immunogenicity of a cluster specific immunotherapy in children with bronchial asthma and mite allergy. Int Arch Allergy Immunol 148: 251-260.

8. Zhang L, Wang C, Han D, Wang X, Zhao Y, et al. (2009) Comparative study of cluster and conventional immunotherapy schedules with dermatophagoides pteronyssinus in the treatment of persistent allergic rhinitis. Int Arch Allergy Immunol 148: 161-169.

9. Pfaar O, Klimek L, Fischer I, Sieber J, Amoroso S, et al. (2009) Safety of two cluster schedules for subcutaneous immunotherapy in allergic rhinitis or asthma patients sensitized to inhalant allergens. Int Arch Allergy Immunol 150: 102-108.

10. Tabar Al, Echechipía S, García BE, Olaguibel JM, Lizaso MT, et al. (2005) Double-blind comparative study of cluster and conventional immunotherapy schedules with dermatophagoides pteronyssinus. J Allergy Clin Immunol 116: 109-118.

11. Roger A, Depreux N, Jurgens Y, Heath MD, Garcia G, et al. (2014) A novel and well tolerated mite allergoid subcutaneous immunotherapy: Evidence of clinical and immunologic efficacy. Immun Inflamm Dis 2: 92-98.

12. Tabar Al, Fernandez-Tavora L, Alonso R, Castillo R, Cistero-Bahima A, et al (2004) Tolerance of a cluster schedule with a house dust mite extract quantified in mass units: Multicentre study. J Investig Allergol Clin Immunol 14: 193-197.
13. Hernandez N, Ibero M, Ridao M, Artigas R, Viñas M, et al. (2011) Safety of specific immunotherapy using a depigmented and polymerised extract of dermatophagoides pteronyssinus in children under five years of age. Allergol Immunopathol (Madr) 39: 267-270.

14. Nieto Garcia A, Nevot Falco S, Carrillo Díaz T, Cumplido Bonny JA, Izquierdo Calderón JP, et al. (2013) Safety of cluster specific immunotherapy with a modified high-dose house dust mite extract. Eur Ann Allergy Clin Immunol 45: 78-83.

15. Colas C, Monzón S, Venturini M, Lezaun A (2006) Double-blind, placebocontrolled study with a modified therapeutic vaccine of salsola kali (russian thistle) administered through use of a cluster schedule. J Allergy Clin Immunol 117: 810-816.

16. Cox L, Larenas-Linnemann D, Lockey RF, Passalacqua G (2010) Speaking the same language: The world allergy organization subcutaneous immunotherapy systemic reaction grading system. J Allergy Clin Immunol 125: 569-574.

17. R Development Core Team (2012) R Core R: A language and environment for statistical computing

18. Yoav Benjamini and Yosef Hochberg (1995) Controlling the false discovery rate: A practical and powerful approach to multiple testing. J R Stat Soc B (Methodological) 57: 289-300.

19. (2015) Global initiative for asthma: global strategy for asthma management and prevention.

20. Douglas RM, Paton JC, Duncan SJ, Hansman DJ (1983) Antibody response to pneumococcal vaccination in children younger than five years of age. $J$ Infect Dis 148: 131-137.

21. Ayumi Mugitani, Kazuya Ito, Shin Irie, Takashi Eto, Motoki Ishibashi, et al. (2014) Immunogenicity of the trivalent inactivated influenza vaccine in young children less than 4 years of age, with a focus on age and baseline antibodies. Clin Vaccine Immunol 21: 1253-1260.

22. Stritzke Al, Eng PA (2013) Age-dependent sting recurrence and outcome in immunotherapy-treated children with anaphylaxis to hymenoptera venom. Clin Exp Allergy 43: 950-955.

23. Alice Kohli-Wiesner, Lisbeth Stahlberger, Christian Bieli, Tamar Stricker, Roger Lauener, et al. (2012) Induction of specific immunotherapy with hymenoptera venoms using ultrarush regimen in children: Safety and tolerance. J Allergy (Cairo) 2012: 790910.

24. Li MR, Wang XN, Jiang HD, Wang QY, Li YC, et al. (2012) Analysis of adverse reactions induced by subcutaneous immunotherapy against dust mite allergy in 234 cases with allergic rhinitis and asthma. Zhonghua $\mathrm{Er} \mathrm{Ke}$ Za Zhi 50: 726-731.

25. García-Ara C, Pedrosa M, Belver MT, Martín-Muñoz MF, Quirce S, et al. (2013) Efficacy and safety of oral desensitization in children with cow's milk allergy according to their serum specific ige level. Ann Allergy Asthma Immunol 110: 290-294.

26. Tabar Al, Muro MD, García BE, Alvarez MJ, Acero S, et al. (1999) Dermatophagoides pteronyssinus cluster immunotherapy. A controlled trial of safety and clinical efficacy. J Investig Allergol Clin Immunol 9: 155-164.

27. Lou W, Wang C, Wang Y, Han D, Zhang L (2012) Responses of cd4 (+) cd25 $(+)$ foxp3 $(+)$ and il-10-secreting type i t regulatory cells to cluster-specific immunotherapy for allergic rhinitis in children. Pediatr Allergy Immunol 23: 140-149. 